\title{
Acute Effects of Tissue Flossing Around the Upper Thigh on Neuromuscular Performance: A Study Using Different Degrees of Wrapping Pressure
}

\author{
Matjaž, Vogrin ${ }^{1,2}$, Miloš Kalc ${ }^{1}$, and Teja Ličen ${ }^{1}$ \\ ${ }^{1}$ University of Maribor, Faculty of Medicine, Institute of Sports Medicine \\ ${ }^{2}$ University Medical Centre Maribor, Department of Orthopaedics
}

May 5, 2020

\begin{abstract}
Context: It has been recently demonstrated that tissue flossing around the ankle joint can be effectively used to improve ankle range of motion, jump and sprint ability. However, there is a lack of studies investigating the acute effects of tissue flossing applied around the thigh. Objective: The study aimed to investigate the acute effects of tissue flossing, and the degree of floss band pressure, around the upper-thigh on Active straight leg raise test, knee extensor and flexor maximum voluntary contraction and associated thigh muscle tensiomyography parameters. Design: Cross-over design in 3 distinct sessions. Setting: University laboratory. Participants: 19 recreationally trained volunteers (age $23.78 \pm 4.85$ ). Intervention: Active knee extension and flexion performed for 3 sets of 2 minutes (2 minutes rest between sets with wrapped upper-thigh. Individualized wrapping pressures were applied to create conditions of high (HIGH) and moderate (LOW) vascular occlusion, while a loose band application served as a control condition (CON). Main Outcome Measures: Participants were assessed for Active straight leg raise test (ASLR), tensiomyography displacement (Dm) and contraction time (Tc) for rectus femoris (RF), vastus medialis $(\mathrm{VM})$ and biceps femoris (BF) muscles, maximum voluntary contractions for knee extensors (extMVC) and flexors (flexMVC) for pre (PRE), after (POST) and 30 minutes after (POST30) applying the floss band. There was a statistically significant increase in extMVC and a significant shortening in rectus femoris Tc for the LOW condition, which was associated with small to medium effects in favor of the LOW condition. There were no statistically significant changes observed between CON and HIGH conditions. The ASLR test was unaffected regardless of intervention. Conclusions: The results of this study suggest that tissue flossing around the upper-thigh might have a localized as well as pressure-sensitive response, thereby improving neuromuscular function of the knee extensors.
\end{abstract}

\section{Introduction}

Tissue flossing has become an increasingly popular technique among physiotherapists and strength and conditioning specialists, mostly used as a rehabilitation or performance enhancing tool ${ }^{1}$. The method consists of tightly wrapping part of a limb or a joint with a 1 to $2 \mathrm{~mm}$ thick elastic band, causing partial vascular occlusion of the blood flow distal to the wrapped area.

Despite the popularity of this method, the acute effects of tissue flossing on neuromuscular functions are still poorly understood; moreover, at the time of writing this paper, there was limited scientific evidence underlying the efficiency of this method. The majority of published studies have investigated the effects of tissue flossing applied to the ankle joint, which resulted in increased ankle range of motion (ROM) ${ }^{2}$, increased jump and sprinting ability ${ }^{1,3}$. On the other hand, only two peer-reviewed studies have investigated the effects of tissue flossing around a limb (thigh and upper-arm). Both the aforementioned studies investigated the 
effects of tissue flossing as a treatment for delayed onset of muscle soreness (DOMS), leading to conflicting results: in the study conducted by ${ }^{4}$, tissue flossing resulted in lower effectiveness than other gold standard methods to alleviate the effects of DOMS. However, the authors advocated that tissue flossing is a valid, practical and cost-effective method. In contrast, ${ }^{5}$ applied three sets of two-minute tissue flossing to the upper-thigh region after an exercise-induced muscle damaging protocol, finding that tissue flossing has no effects on DOMS reduction.

Although there are no available studies directly comparing tissue flossing with ischemic preconditioning/blood flow restriction (BFR) training, there are similarities between the methods ${ }^{1}$. Similar to tissue flossing, BFR training consists of complete occlusion of venous and partial occlusion of arterial blood flow to a limb using an elastic wrap ${ }^{6}$ or a specialized inflatable cuff ${ }^{7}$. There is strong evidence in the literature showing the positive effects of low-load BFR training on chronic neuro-muscular adaptations ${ }^{8,9,10,11}$. On the other hand, there is evidence in the literature that single low-load BFR training sessions acutely decrease neuromuscular performance ${ }^{12,13,14}$, although ${ }^{15}$ failed to find such differences during a multi-set resistance exercise leading to volitional failure in diverse conditions of systemic hypoxia, BFR, or normoxia. Divergence in the literature arises from differences in protocols, in particular, in the use of different restrictive pressures. Some authors ${ }^{16}$ have proposed cuff pressure individualization to achieve more reliable results. Indeed, ${ }^{17}$ and ${ }^{18}$ demonstrated that different relative blood flow occlusions result in different neuromuscular impairments.

To our knowledge, there are no studies in the literature investigating the effect of tissue flossing around a limb on neuromuscular performance. Moreover, a fixed apriori-defined high wrapping pressure (approx. $>180 \mathrm{mmHg}$ ) was used in the majority of previous tissue flossing studies ${ }^{1,2,3,19}$. Therefore, the aim of this study was to investigate the acute effects of tissue flossing applied over the upper-thigh region by using different degrees of wrapping pressure on Active straight leg raise test, maximum voluntary contraction, and the contractile characteristics of knee extensors and flexors assessed using tensiomyography.

\section{Methods}

\section{Study design}

The present study followed a cross-over repeated measures design. Each subject was exposed to three similar intervention protocols, one per visit. At each visit, following warm-up and pretest assessments, the upper-thigh of the dominant leg was wrapped using a rubber elastic band (floss band), with subjects being instructed to perform active knee extension and flexion movements. The pressure used to wrap the band was individualized to each subjects' thigh circumference and was different on each visit according to the selected protocol (CON, LOW, HIGH). During each visit, comprising pre (PRE), immediately after (POST) and 30 minutes after (POST30) applying the floss band, participants performed several lower-leg assessments in the following order: i) Active straight leg raise test (ASLR); ii) TMG of the rectus femoris (RF), vastus medialis (VM) and biceps femoris (BF) muscles; iii) maximum voluntary contractions of knee extensors and flexors - MVC (see Figure 1). There were at least 48-hours of delay between consecutive visits to avoid between-session influences. 


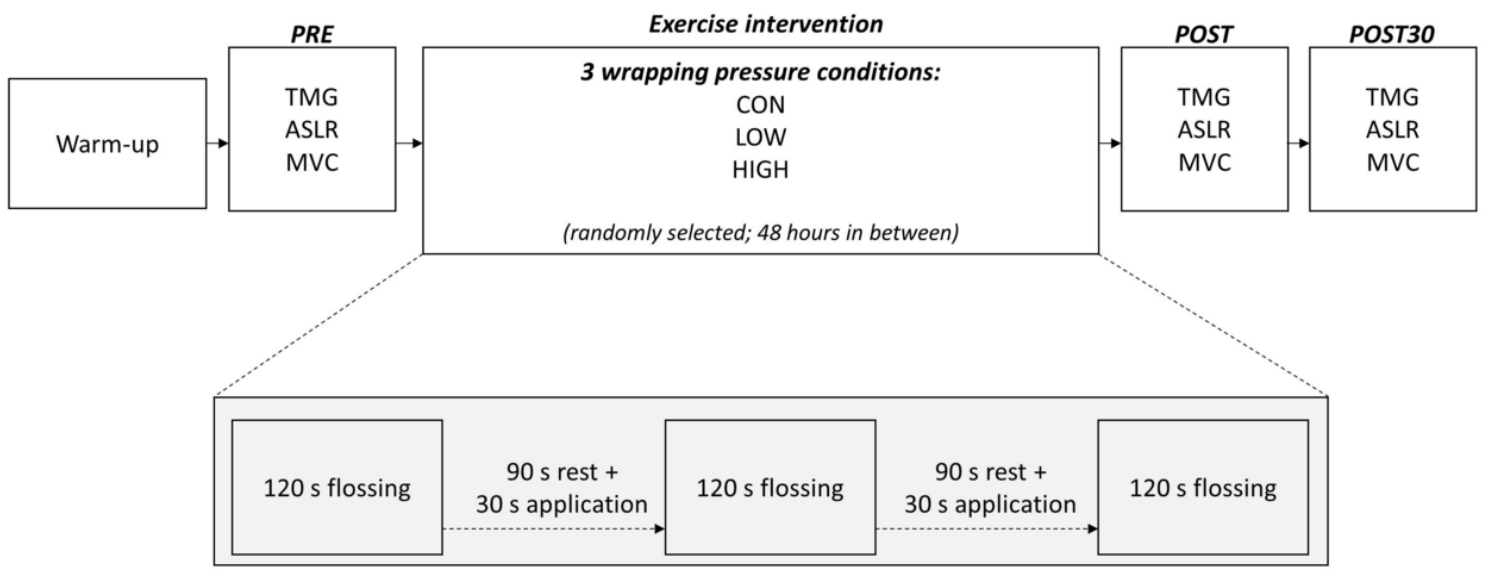

Figure 1: Graphical representation of the study design. TMG - Rectus Femoris muscle and Biceps Femoris muscle tensiomyography assessment; ASLR - active straight leg raise test; MVC - knee extensors and flexors maximum voluntary contraction assessment.

\section{Participants}

19 recreationally trained volunteers (Gender: 14 men, 5 women, Age: $23.78 \pm 4.85$ years, Fat mass: $20.34 \%$ \pm 6.43 , Muscle mass: $55.28 \% \pm 9.58)$ participated in this study. All participants regularly took part in physical activity sessions (approx. 3 times per week) and were free of any locomotor, nerve, or known cardiovascular and metabolic diseases. All subjects were instructed to avoid heavy strength training (heavy weightlifting, plyometric drills, intensive interval training) 24 hours before each assessment. The volunteers were familiarized with the experimental procedure and their voluntary cooperation confirmed by written informed consent on the first testing day. The study was approved by the Research Ethics Committee of the [** Institution name - it will be filled after review]. All procedures were performed according to the Declaration of Helsinki.

\section{Preliminary procedures}

Upon their arrival at the laboratory for the first visit, the subjects' weight and body composition were measured. The subjects verbally self-selected their dominant leg, but those who were unsure undertook a dominant leg assessment task where they were slightly pushed forward from a standing position, and the dominant leg determined according to which leg stepped forward to accommodate the movement. The distances between the great trochanter and the lateral condyle of the femur for each dominant leg (15 left, 4 right) were measured using a measuring tape (20 $\mathrm{mm}$ wide, $1 \mathrm{~mm}$ accuracy). From the obtained distance, the mid and upper-third thigh-length were thus defined and marked with a medical skin marker. Thigh circumference was recorded at the upper-third mark, with the mid-thigh mark serving as the starting point for the floss band application. 


\section{Warm-up routine}

Prior to each pretest, participants performed a warm-up routine consisting of 5-minute upright cycling on a cycle ergometer (Lode, BV The Netherlands) at an intensity of $1 \mathrm{~W} / \mathrm{Kg}$ of body weight at a cadence of 80 RPM.

\section{Floss band application}

Following pretests, participants were instructed to lie prone on a (standard) therapeutic table (with knee of the dominant leg slightly flexed), while the floss band comprising a thick latex elastic band (Medical Flossing Band, Germany, $1.3 \mathrm{~mm}$ thick, $50 \mathrm{~mm}$ wide, $150 \mathrm{~mm}$ long) was wrapped around the upper-thigh of the dominant leg. A simple thigh-bandaging technique was used: with its origin at the mid-thigh mark, the elastic band was first pulled and then wrapped in a circular motion around the thigh moving proximally towards the hip in a progressive way. Each subsequent wrap overlapped the previous by approx. 50\%, before fixing the last part of the band (approx. $5 \mathrm{~cm}$ ) underneath the final wrap. When fully applied, the floss band covered approx. $15 \mathrm{~cm}$ of the thigh. The pressure used to wrap the thigh was individualized to the subjects' thigh circumference and differed for each visit according to the selected protocol (CON, LOW, HIGH). Once the floss band was applied, participants were instructed to sit on the edge of the therapeutic table, with $\frac{3}{4}$ of the thigh supported by the therapeutic table, feet unsupported, performing slow continuous active knee extension ( 2 seconds) and flexion ( 2 seconds) ranging from a bent knee position $\left(90^{\circ}\right)$ to knee fully extended $\left(0^{\circ}\right)$. After $120 \mathrm{~s}$ the floss band was unwrapped, and participants were allowed to rest for 120 $\mathrm{s}$. In the rest period, the subjects were instructed to lie still on their backs on the therapeutic table, while the re-application of floss band was carried out in the last $30 \mathrm{~s}$ of the rest period. This procedure was repeated three times ( 3 sets of $120 \mathrm{~s}$ flossing with active motion followed by $90 \mathrm{~s}$ of rest and $30 \mathrm{~s}$ of re-application). 

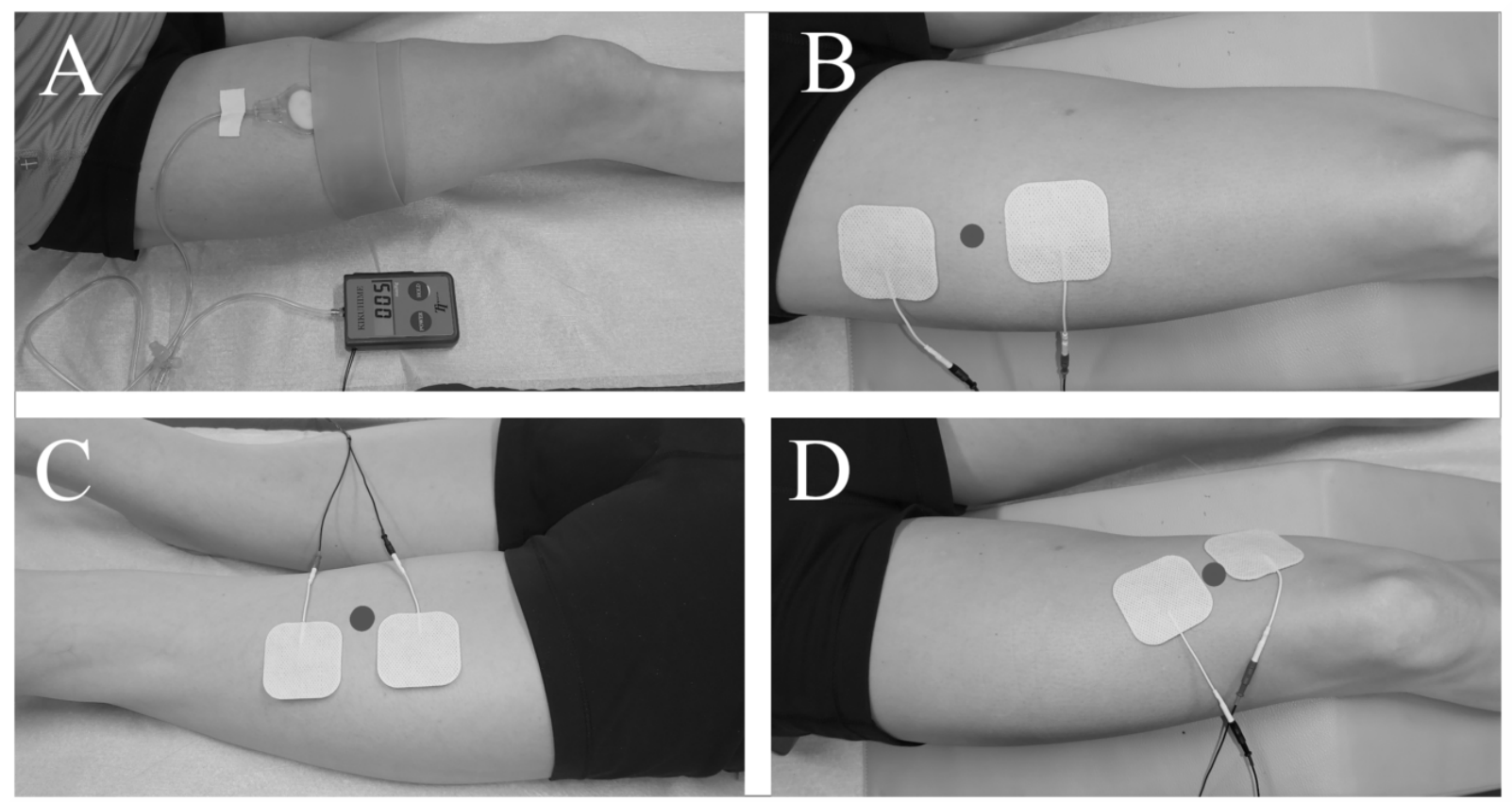

Figure 2: Photographic representation of floss band, pressure sensor and TMG placement. A - Pressure sensor partially wrapped under floss band; B - electrical stimulation and TMG sensor probe placement on the RF muscle; $\mathrm{C}$ - electrical stimulation and TMG sensor probe placement on the BF muscle; D - electrical stimulation and TMG sensor probe placement on the VM muscle.

\section{Kikuhime Pressure Control Sensor measurement}

A flat balloon-like pressure control sensor (35 mm in diameter; Kikuhime, TT Meditrade, Sor $\varnothing$, Denmark) was used to control the pressure exerted by the floss band to the wrapped thigh. This device represents a validated $(\mathrm{ICC}=0.99, \mathrm{CV}=1.1 \%)$ and reliable $(\mathrm{CV}=4.9 \%)$ tool to be used in the sports medicine setting ${ }^{20}$. The Kikuhume pressure control sensor was fixed with a tape patch over the tube of the sensor, approx. $4 \mathrm{~cm}$ above the mid-thigh mark on the RF. To ensure the precise pressure measurement of the pressure, the tube attaching the sensor to the digital display was set towards the hip while the sensor itself was set towards the floss band and progressively compressed by the band application around the thigh (Figure 2A). We ensured that the measuring surface of the sensor was always fully covered by the floss band.

\section{Thigh wrapping pressure}

Three intervention conditions (one per visit) were induced by applying individualized thigh wrapping pressures (CON, LOW, HIGH). A $20 \mathrm{mmHg}$ pressure was used for all participants to ensure the control condition (CON). Pressures for LOW and HIGH conditions were individualized following the adapted BFR pressure guidelines proposed by ${ }^{17}$, which were suggested to produce $40 \%$ and $60 \%$ vascular occlusion, respectively (Table 1) 

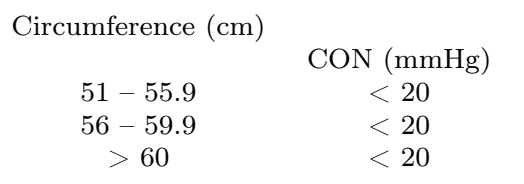
Target pressure
LOW (mmHg)
100
120
140

HIGH (mmHg)

$150 \quad 2$

180

$210 \quad 12$

Table 1: Wrapping pressures based on thigh circumference adapted from ${ }^{17}$. Abbreviations: CON - Control intervention; LOW - Low pressure intervention; HIGH - High pressure intervention; Subjects - number of subjects included in each thigh circumference group.

\section{Assessment procedures}

All described procedures were performed on the dominant leg only.

\section{Active straight leg raise test}

The Active straight leg raise test (ASLR) was assessed using a digital goniometer (BTS Bioengineering, Italy) placed on the front part of the mid-tibial plateau of the dominant leg and fixed in position with an elastic band. Participants were instructed to lay supine on a (standard) therapeutic table, with arms alongside the body. Each was instructed to flex the hip slowly (full raise in approx. $2 \mathrm{~s}$ ), while maintaining a fully extended knee. Once they reached the maximal active range of motion, they held the final position for approximately 1 second before slowly (approx. $2 \mathrm{~s}$ ) returning to the starting position. The contralateral leg remained straight and in contact with the table at all times, while the researcher stabilized the pelvis by holding the anterior superior iliac spine down. The procedure was repeated twice at each measuring time (PRE, POST, and POST30), and the maximum ASLR angle $\left(^{\circ}\right)$ at each measuring time was recorded for further analysis.

\section{Tensiomiography (TMG)}

Tensiomyography (TMG), which comprises a non-invasive probe-like digital displacement transducer (TMGBMC Ltd, Ljubljana, Slovenia), was used to evaluate the muscle contractile properties of RF, VL, and BF. For evaluating the RF and VL parameters, subjects were instructed to lie in a supine position on the therapeutic table, with a triangular cushion placed below their knee joint to support approximately $30^{\circ}$ of knee flexion. For the BF assessment, subjects were asked to turn over to a prone position with their head turned to one side and arms alongside their body. A foam cushion placed underneath the ankle supported five degrees of plantarflexion. The TMG sensor was placed in contact with the skin, perpendicular to the tangential plane of the muscle belly for the selected muscle, held in place by a tripod-mounted mechanical arm exerting minimal pressure (approx. $1.5 \times 10^{-2} \mathrm{~N} / \mathrm{mm}^{2}-$ manufacturers data). Correct probe placement was determined by instructing the subject to slightly contract the thigh muscles (knee extensors for RF and VL; knee flexors for BF), which allowed the researcher to visually and manually pulsate the thickest area of the muscle belly. The probe was additionally adjusted, if needed, to obtain the greatest mechanical response when the muscle was electrically stimulated.

TMG evaluation was performed by electrically stimulating the selected muscles. Two self-adhesive $5 \times 5 \mathrm{~cm}$ electrodes were placed equidistant from the TMG probe sensor and in line with the muscle fibers (Figure $2 \mathrm{~B}, 2 \mathrm{C}$, and $2 \mathrm{D})$. The position of the electrodes and the probe sensor were accurately marked with a medical skin marker to avoid alterations in muscle response during within days assessments ${ }^{21}$ and to ensure accurate repositioning.

A constant current electrical stimulator (TMG-BMC Ltd, Ljubljana, Slovenia) was used to deliver a single 1 ms wide stimulation pulse to elicit a mechanical muscle response. The highest mechanical response for each muscle was determined at baseline: a small initial stimulation amplitude (30 mA) was adopted at first and 
progressively increased by $10 \mathrm{~mA}$ increments until the mechanical response reached its maximum. Electrical stimuli were evoked 10 seconds apart to avoid the phenomenon of post-activation potentiation or fatigue. As proposed by ${ }^{22}$, the two highest mechanical responses and related stimulation amplitudes were recorded and saved during each testing. Stimulation amplitudes used to elicit the highest mechanical response at baseline were reused later in the protocol when TMG was assessed after floss band application (POST, POST30). The highest mechanical responses were generally elicited at stimulation amplitudes between $60 \mathrm{~mA}$ and 90 $\mathrm{mA}$.

Every mechanical response was stored and displayed in the TMG software and was graphically represented as the change in muscle displacement over time - displacement curve., The following parameters can be computed from every displacement curve: contraction time ( $\mathrm{Tc}$ - expressed in ms), maximal displacement of the muscle belly ( $\mathrm{Dm}$ - expressed in $\mathrm{mm}$ ) delay time ( $\mathrm{Td}$ - expressed in $\mathrm{ms}$ ), sustained time ( $\mathrm{Ts}$ - expressed in $\mathrm{ms}$ ) and half-relaxation time ( $\mathrm{Tr}$ - expressed in $\mathrm{ms}$ ). Since inter-rater ${ }^{21}$ and between-day reliability ${ }^{22}$ of Tc and Dm parameters have been shown to be the most reliable and sufficient for comparison, these two parameters have been calculated and used for further analysis in this study.

\section{Maximum voluntary contraction (MVC) assessment}

Isometric maximum voluntary contractions (MVC) of knee extensors and flexors were assessed using a Kineo ${ }^{\circledR}$ dynamometer (GLOBUS, Codognè, Italy). To assess MVC of knee extensors (extMVC), participants were instructed to sit on a leg extension machine with their hips flexed at $80^{\circ}\left(0^{\circ}\right.$ hip fully extended) and additionally secured with padded straps around the pelvis. The medial epicondyle of the femur was aligned with the extension machine's axis of rotation, while the knee was flexed at $60^{\circ}\left(0^{\circ}\right.$ knee fully extended $)$ and a padded lever arm was placed on the tibia just above the anterior part of the ankle joint.

For knee flexion MVC (flexMVC), participants were instructed to stand facing the Kineo ${ }^{\circledR}$ machine, pressing the pelvis into the pad in front of them, which prevented any compensatory hip flexion or extension. They were instructed to firmly hold the vertical bar (fixed on the machine) in front of them to further stabilize their body. The participants stood on their non-dominant leg on a wooden step (ranging from 10-25 $\mathrm{cm}$ in height) to provide the ground clearance needed to allow optimal knee alignment with the machine's axis of rotation. The dominant leg was fixed at a position of $30^{\circ}$ knee flexion, while the padded lever arm was placed on the tibia just above the posterior part of the ankle joint.

Participants were allowed to perform 3 submaximal isometric knee extensions of the duration of $5 \mathrm{~s}$ to become familiarized with the testing procedure. The participants were instructed to perform 2 maximal isometric contractions of $5 \mathrm{~s}$ duration each for knee extension and flexion respectively. A $30 \mathrm{~s}$ rest was allowed between trials. Participants were verbally encouraged to perform the tests with maximal effort during testing. The maximum peak torque from the two trials was taken for further analysis. 


\begin{tabular}{|c|c|c|c|c|c|c|c|c|c|}
\hline \multirow{2}{*}{$\begin{array}{l}\text { Assess- } \\
\text { ment }\end{array}$} & \multicolumn{3}{|c|}{$\mathrm{CON}$} & \multicolumn{3}{|c|}{ LOW } & \multicolumn{3}{|c|}{ HIGH } \\
\hline & PRE & POST & POST30 & PRE & POST & POST30 & PRE & POST & POST30 \\
\hline ALSR & 81.74 & 80.72 & 78.76 & 79.25 & 80.33 & 79.42 & 82.18 & 82.62 & 81.74 \\
\hline$\left(^{\circ}\right)$ & \pm 10.3 & \pm 9.32 & $\begin{array}{c} \pm \\
10.91\end{array}$ & \pm 9.97 & $\begin{array}{c} \pm \\
11.77\end{array}$ & $\begin{array}{c} \pm \\
12.71\end{array}$ & \pm 8.36 & \pm 9.99 & $\begin{array}{c} \pm \\
11.25\end{array}$ \\
\hline $\begin{array}{l}\text { extMVC } \\
(\mathrm{Nm})\end{array}$ & $\begin{array}{l}424.88 \\
\quad \pm\end{array}$ & $\begin{array}{l}428.23 \\
\quad \pm\end{array}$ & $\begin{array}{c}433.65 \\
\quad \pm\end{array}$ & $\begin{array}{l}421.37 \\
\quad \pm\end{array}$ & $\begin{array}{l}445.94 \\
\quad \pm\end{array}$ & $\begin{array}{l}441.76 \\
\quad \pm\end{array}$ & $\begin{array}{l}418.47 \\
\quad \pm\end{array}$ & $\begin{array}{l}429.16 \\
\quad \pm\end{array}$ & $\begin{array}{l}420.38 \\
\quad \pm\end{array}$ \\
\hline & 96.87 & 102.07 & 105.68 & 102.22 & 104.3 & 105.52 & 102.47 & 101.43 & 106.11 \\
\hline flexMVC & 212.41 & 216.08 & 216.44 & 215.82 & 220.26 & 211.59 & 203.33 & 211.07 & 207.87 \\
\hline$(\mathrm{Nm})$ & $\begin{array}{c} \pm \\
79.16\end{array}$ & \pm 78.0 & \pm 75.4 & $\begin{array}{c} \pm \\
80.85\end{array}$ & $\begin{array}{c} \pm \\
75.12\end{array}$ & $\begin{array}{c} \pm \\
76.78\end{array}$ & $\begin{array}{c} \pm \\
68.31\end{array}$ & $\begin{array}{c} \pm \\
74.69\end{array}$ & $\begin{array}{c} \pm \\
68.53\end{array}$ \\
\hline Tc_- & 26.49 & 25.38 & 24.97 & 26.75 & 24.41 & 25.82 & 25.19 & 24.95 & $26.2 \pm$ \\
\hline $\begin{array}{l}\mathrm{R} \overline{\mathrm{F}} \\
(\mathrm{ms})\end{array}$ & \pm 5.44 & \pm 4.18 & \pm 4.55 & \pm 4.2 & \pm 3.59 & \pm 3.02 & \pm 2.7 & \pm 3.19 & 3.54 \\
\hline $\begin{array}{c}\text { Tc_BF } \\
(\overline{m s})\end{array}$ & $\begin{array}{c}27.45 \\
\pm 9.92\end{array}$ & $\begin{array}{c}28.45 \\
\pm 9.06\end{array}$ & $\begin{array}{c}28.07 \\
\pm \\
11.31\end{array}$ & $\begin{array}{c}24.84 \\
\pm 6.64\end{array}$ & $\begin{array}{c}27.08 \\
\pm \\
11.19\end{array}$ & $\begin{array}{c}28.7 \pm \\
10.58\end{array}$ & $\begin{array}{c}26.17 \\
\pm 7.13\end{array}$ & $\begin{array}{c}24.89 \\
\pm 7.34\end{array}$ & $\begin{array}{c}26.68 \\
\pm 7.22\end{array}$ \\
\hline Tc_- & $23.7 \pm$ & 23.59 & 24.46 & 24.12 & 24.21 & 24.35 & 24.24 & 23.16 & 24.34 \\
\hline $\begin{array}{l}\mathrm{V} \overline{\mathrm{M}} \\
(\mathrm{ms})\end{array}$ & 2.4 & \pm 9.06 & \pm 2.55 & \pm 6.64 & \pm 1.76 & $\begin{array}{c} \pm \\
10.58\end{array}$ & \pm 2.28 & \pm 2.38 & \pm 2.55 \\
\hline $\begin{array}{c}\mathrm{Dm}_{-}^{-} \\
\mathrm{RF}^{-} \\
(\mathrm{mm})\end{array}$ & $\begin{array}{c}6.71 \pm \\
2.33\end{array}$ & $\begin{array}{c}7.19 \pm \\
2.88\end{array}$ & $\begin{array}{c}6.73 \pm \\
2.47\end{array}$ & $\begin{array}{c}6.93 \pm \\
2.37\end{array}$ & $\begin{array}{c}6.49 \pm \\
2.3\end{array}$ & $\begin{array}{c}7.24 \pm \\
2.53\end{array}$ & $\begin{array}{c}6.69 \pm \\
2.05\end{array}$ & $\begin{array}{c}6.72 \pm \\
2.28\end{array}$ & $\begin{array}{c}7.52 \pm \\
2.89\end{array}$ \\
\hline $\begin{array}{c}\mathrm{Dm}_{-}^{-} \\
\mathrm{BF} \\
(\mathrm{mm})\end{array}$ & $\begin{array}{c}5.51 \pm \\
3.67\end{array}$ & $\begin{array}{c}5.88 \pm \\
3.45\end{array}$ & $\begin{array}{c}5.15 \pm \\
3.23\end{array}$ & $\begin{array}{c}4.52 \pm \\
2.89\end{array}$ & $\begin{array}{c}4.59 \pm \\
2.74\end{array}$ & $\begin{array}{c}4.81 \pm \\
3.06\end{array}$ & $\begin{array}{c}4.86 \pm \\
2.39\end{array}$ & $\begin{array}{c}4.26 \pm \\
2.34\end{array}$ & $\begin{array}{c}4.96 \pm \\
2.78\end{array}$ \\
\hline $\begin{array}{c}\mathrm{Dm}_{-}^{-} \\
\mathrm{VM} \\
(\mathrm{mm})\end{array}$ & $\begin{array}{c}8.02 \pm \\
2.32\end{array}$ & $\begin{array}{c}8.42 \pm \\
1.92\end{array}$ & $\begin{array}{c}8.1 \pm \\
1.52\end{array}$ & $\begin{array}{c}7.51 \pm \\
1.93\end{array}$ & $\begin{array}{c}7.67 \pm \\
1.8\end{array}$ & $\begin{array}{c}8.08 \pm \\
1.64\end{array}$ & $\begin{array}{c}8.1 \pm \\
1.83\end{array}$ & $\begin{array}{c}8.17 \pm \\
1.77\end{array}$ & $\begin{array}{c}8.05 \pm \\
1.67\end{array}$ \\
\hline
\end{tabular}

Table 2: Descriptive statistics (mean \pm SD). Abbreviations: CON - control intervention; LOW Low-wrapping pressure intervention; HIGH - High-wrapping pressure intervention; PRE - assessment before intervention; POST - assessment after intervention; POST30 - assessment 30 minutes after intervention; ALSR - Active straight leg raise test; extMVC - knee extensors maximum voluntary contractions; flexMVC - knee flexors maximum voluntary contractions TcRF - rectus femoris muscle contraction time; TcBF biceps femoris muscle contraction time; TcVM -vastus medialis muscle contraction time; DmRF -rectus femoris muscle displacement; DmBF -biceps femoris muscle displacement; DmVM -vastus medialis muscle displacement.

\section{Statistical analysis}

Data was analyzed using the $\mathrm{R}$ (version 3.5.3) programming language. Normal distribution was verified using the Shapiro-Wilk test for small samples across all parameters. A 3x3 two-way repeated-measures ANOVA using the Afex package was performed to determine the effect of different treatments (CON, LOW, HIGH) over time (PRE, POST, POST30) on all measured variables. The assumption of sphericity was assessed using the Mauchly test. Whenever the assumption of sphericity was violated, the degrees of freedom were corrected using the Greenhouse-Geisser correction (GGe). Post-hoc tests were performed as pairwise comparisons using Tukey's adjustment within the Emmeans package to determine the differences between single treatments at different time points. Standardized changes in the mean of each measure were used to assess magnitudes of 
effects and were calculated using Cohen's d and then interpreted using thresholds of $0.2,0.5,0.8$ for small, moderate and large, respectively ${ }^{23}$. An effect size of \pm 0.2 was considered the smallest worthwhile effect with an effect size of $<0.2$ considered to be trivial. The effect was considered unclear if its $95 \%$ confidence interval overlapped the thresholds for small positive and small negative effects ${ }^{23}$. Statistical significance was set at $\mathrm{p}<0.05$ for all analyses.

\section{Results}

Descriptive statistics (mean and SD) for ASLR, MVC, and TMG data are given in Table 2. All observed variables were normally distributed. Cohen's d effect sizes for comparisons of all post measures (POST, POST30) to pretest values are given in Table 3.

\section{ASLR analysis}

The analysis of ASLR data revealed there were no significant differences in the main effect of time $(\mathrm{F}(2,36)$ $=2.02, \mathrm{p}=0.146)$; type of intervention $(\mathrm{F}(2,36)=1.58, \mathrm{p}=0.238)$; or interaction between the type of intervention and time $(\mathrm{F}(4,72)=1.31, \mathrm{p}=0.272)$. There were small to medium benefits associated with the LOW condition compared to CON and small benefits associated with the HIGH condition compared to CON. There were unclear benefits associated with the LOW condition compared to HIGH.

\section{MVC analysis}

The analysis of extMVC data revealed statistically significant differences in the main effect of time $(\mathrm{F}(2,36)$ $=5.05, \mathrm{p}=0.012)$; however, there were no significant differences in the main effect of type of intervention $(\mathrm{F}(2,36)=1.16, \mathrm{p}=0.324)$; or interaction between the type of intervention and time $(\mathrm{F}(4,72)=1.92$, $\mathrm{p}=0.116)$. Post-hoc tests revealed significant statistical differences for the LOW condition between PRE and POST measurements $(\mathrm{t}(107)=2.50, \mathrm{p}=0.001)$ and between PRE and POST30 measurements $(\mathrm{t}(107)$ $=2.08, \mathrm{p}=0.011)$. There were small to medium benefits associated with the LOW condition compared to CON and small benefits associated with the HIGH condition compared to CON. There were medium benefits associated with the LOW condition compared to HIGH. The analysis of flexMVC data revealed no statistically significant differences in the main effect of time $(\mathrm{F}(2,36)=1.32, \mathrm{p}=0.278)$; type of intervention $(\mathrm{F}(2,36)=1.70, \mathrm{p}=0.196)$; or interaction between the type of intervention and time $(\mathrm{F}(4,72)=0.80$, $\mathrm{p}=0.530)$. There were unclear to small benefits associated with the LOW condition compared to CON and unclear benefits associated with the HIGH condition compared to CON. There were unclear to small benefits associated with the HIGH condition compared to LOW.

\section{TMG analysis}

The analysis of Tc_RF data revealed statistically significant differences in the main effect of time $(\mathrm{F}(2,36)$ $=4.62, \mathrm{p}=0.016)$; however, there were no significant differences in the main effect of type of intervention $(\mathrm{F}(2,36)=0.04, \mathrm{p}=0.957)$; or interaction between the type of intervention and time $(\mathrm{F}(4,72)=1.69$, $\mathrm{p}=0.160)$. Post-hoc tests revealed significant statistical differences for the LOW condition between PRE and POST measurements $(\mathrm{t}(104)=2.85, \mathrm{p}=0.014)$. There were small benefits associated with the LOW condition compared to CON and small to medium benefits associated with the CON condition compared to HIGH. There were medium benefits associated with the LOW condition compared to HIGH. The analysis of Tc_BF data revealed no statistically significant differences in the main effect of time $(\mathrm{F}(2,36)=1.82$, p $=0.1 \overline{7} 6)$; type of intervention $(\mathrm{F}(2,36)=0.64, \mathrm{p}=0.532)$; or interaction between the type of intervention and time $(\mathrm{F}(4,72)=1.60, \mathrm{p}=0.184)$. There were unclear to small benefits associated with the LOW condition compared to CON and unclear to small benefits associated with the HIGH condition compared 
to CON. There were small benefits associated with the HIGH condition compared to LOW. The analysis of Tc_VM data revealed statistically significant differences in the main effect of time $(\mathrm{F}(2,36)=3.28$, $\mathrm{p}=0.049)$; however, there were no significant differences in the main effect of type of intervention $(\mathrm{F}(2$, $36)=3.88, \mathrm{p}=0.681)$; or interaction between the type of intervention and time $(\mathrm{F}(4,72)=1.62, \mathrm{p}$ $=0.176)$. Post-hoc tests revealed significant statistical differences for CON pressures between PRE and POST30 measurements $(\mathrm{t}(107)=2.31, \mathrm{p}=0.046)$. There were small benefits associated with the LOW condition compared to CON and small benefits associated with the CON condition compared to HIGH. There were unclear to small benefits associated with the HIGH condition compared to LOW. The analysis of Dm_RF data revealed no statistically significant differences in the main effect of time $(\mathrm{F}(2,36)=1.48$, $\mathrm{p}=0 . \overline{241})$; type of intervention $(\mathrm{F}(2,36)=0.04, \mathrm{p}=0.956)$; and a tendency towards statistical significant differences in the interaction between the type of intervention and time $(\mathrm{F}(4,72)=1.49, \mathrm{p}=0.213)$. There were medium benefits associated with the LOW condition compared to CON and small benefits associated with the HIGH condition compared to CON. There were small benefits associated with the LOW condition compared to HIGH. The analysis of Dm_BF data revealed no statistically significant differences in the main effect of time $(\mathrm{F}(2,36)=0.12, \mathrm{p}=0.886)$; type of intervention $(\mathrm{F}(2,36)=1.58, \mathrm{p}=0.220)$; and a tendency towards statistical significant differences in the interaction between the type of intervention and time $(\mathrm{F}(4,72)=2.47, \mathrm{p}=0.052)$. There were small benefits associated with the LOW condition compared to CON and medium benefits associated with the HIGH condition compared to CON. There were unclear to medium benefits associated with the HIGH condition compared to LOW. The analysis of Dm_VM data revealed no statistically significant differences in the main effect of time $(\mathrm{F}(2,36)=0.76, \mathrm{p}=0.473)$; type of intervention $(\mathrm{F}(2,36)=0.95, \mathrm{p}=0.393)$; and a tendency towards statistical significant differences in the interaction between the type of intervention and time $(\mathrm{F}(4,72)=1.84, \mathrm{p}=0.131)$. There were small benefits associated with the LOW condition compared to CON and unclear to small benefits associated with the HIGH condition compared to CON. There were trivial to small benefits associated with the LOW condition compared to HIGH. 


\begin{tabular}{|c|c|c|c|c|c|c|}
\hline $\begin{array}{l}\text { Assess- } \\
\text { ment }\end{array}$ & $\begin{array}{c}\Delta \mathrm{LOW}- \\
\Delta \mathrm{CON} \\
\text { effect size } \\
{[95 \% \mathrm{CI}]} \\
\text { POST }\end{array}$ & & $\begin{array}{c}\Delta \text { HIGH }- \\
\Delta \text { CON } \\
\text { effect size } \\
{[95 \% \mathrm{CI}]} \\
\text { POST }\end{array}$ & & $\begin{array}{c}\Delta \text { HIGH }- \\
\Delta \text { LOW } \\
\text { effect size } \\
{[95 \% \mathrm{CI}]} \\
\text { POST }\end{array}$ & \\
\hline & POST & POST30 & POST & POST30 & POST & POST30 \\
\hline ASLR & $\begin{array}{c}0.46 \pm 0.82 \\
\text { small }\end{array}$ & $\begin{array}{l}0.62 \pm 0.59 \\
\text { medium }\end{array}$ & $\begin{array}{c}0.29 \pm 0.69 \\
\text { small }\end{array}$ & $\begin{array}{c}0.49 \pm 0.71 \\
\text { small }\end{array}$ & $\begin{array}{c}-0.12 \pm 0.47 \\
\text { unclear }\end{array}$ & $\begin{array}{c}-0.11 \pm 0.59 \\
\text { unclear }\end{array}$ \\
\hline extMVC & $\begin{array}{c}0.65 \pm 0.78 \\
\text { medium }\end{array}$ & $\begin{array}{l}0.36 \pm 0.7 \\
\text { small }\end{array}$ & $\begin{array}{c}0.23 \pm 0.62 \\
\text { small }\end{array}$ & $\begin{array}{c}-0.21 \pm 0.64 \\
\text { small }\end{array}$ & $\begin{array}{c}-0.63 \pm 0.8 \\
\text { medium }\end{array}$ & $\begin{array}{c}-0.61 \pm 0.66 \\
\text { medium }\end{array}$ \\
\hline flexMVC & $\begin{array}{c}0.04 \pm 0.58 \\
\text { unclear }\end{array}$ & $\begin{array}{c}-0.41 \pm 0.82 \\
\text { small }\end{array}$ & $\begin{array}{c}0.18 \pm 0.53 \\
\text { unclear }\end{array}$ & $\begin{array}{c}0.02071 \\
\text { unclear }\end{array}$ & $\begin{array}{c}0.16 \pm 0.62 \\
\text { unclear }\end{array}$ & $\begin{array}{c}0.37 \pm 0.46 \\
\text { small }\end{array}$ \\
\hline Tc_RF & $\begin{array}{c}-0.32 \pm 0.73 \\
\text { small }\end{array}$ & $\begin{array}{c}0.15 \pm 0.72 \\
\text { unclear }\end{array}$ & $\begin{array}{l}0.29 \pm 0.5 \\
\text { small }\end{array}$ & $\begin{array}{l}0.71 \pm 0.73 \\
\text { medium }\end{array}$ & $\begin{array}{l}0.51 \pm 0.84 \\
\text { medium }\end{array}$ & $\begin{array}{l}0.52 \pm 0.68 \\
\text { medium }\end{array}$ \\
\hline Tc_BF & $\begin{array}{l}0.18 \pm 0.74 \\
\text { unclear }\end{array}$ & $\begin{array}{c}0.47 \pm 0.52 \\
\text { small }\end{array}$ & $\begin{array}{l}-0.42 \pm 0.7 \\
\quad \text { small }\end{array}$ & $\begin{array}{c}-0.02 \pm 0.65 \\
\text { unclear }\end{array}$ & $\begin{array}{c}-0.45 \pm 0.58 \\
\text { small }\end{array}$ & $\begin{array}{c}-0.47 \pm 0.6 \\
\text { small }\end{array}$ \\
\hline $\mathrm{Tc}_{-} \mathrm{VM}$ & $\begin{array}{c}0.14 \pm 0.54 \\
\text { unclear }\end{array}$ & $\begin{array}{c}-0.36 \pm 0.52 \\
\text { small }\end{array}$ & $\begin{array}{c}-0.34 \pm 0.51 \\
\text { small }\end{array}$ & $\begin{array}{c}-0.41 \pm 0.64 \\
\text { small }\end{array}$ & $\begin{array}{c}-0.45 \pm 0.63 \\
\text { small }\end{array}$ & $\begin{array}{c}-0.1 \pm 0.61 \\
\text { unclear }\end{array}$ \\
\hline $\begin{array}{l}\mathrm{Dm} \\
\mathrm{RF}\end{array}$ & $\begin{array}{c}-0.58 \pm 0.67 \\
\text { medium }\end{array}$ & $\begin{array}{c}0.2 \pm 0.65 \\
\text { unclear }\end{array}$ & $\begin{array}{c}-0.32 \pm 0.53 \\
\text { small }\end{array}$ & $\begin{array}{l}0.36 \pm 0.67 \\
\text { small }\end{array}$ & $\begin{array}{c}0.31 \pm 0.58 \\
\text { small }\end{array}$ & $\begin{array}{l}0.21 \pm 0.62 \\
\text { small }\end{array}$ \\
\hline $\mathrm{Dm}_{\mathrm{BF}}^{-}$ & $\begin{array}{c}-0.24 \pm 0.7 \\
\text { small }\end{array}$ & $\begin{array}{c}0.43 \pm 0.69 \\
\text { small }\end{array}$ & $\begin{array}{c}-0.74 \pm 0.94 \\
\text { medium }\end{array}$ & $\begin{array}{l}0.31 \pm 0.8 \\
\text { small }\end{array}$ & $\begin{array}{c}-0.51 \pm 0.62 \\
\text { medium }\end{array}$ & $\begin{array}{c}-0.16 \pm 0.7 \\
\text { unclear }\end{array}$ \\
\hline $\mathrm{Dm}^{\mathrm{VM}}{ }^{-}$ & $\begin{array}{c}-0.19 \pm 0.59 \\
\text { unclear }\end{array}$ & $\begin{array}{l}0.35 \pm 0.7 \\
\text { small }\end{array}$ & $\begin{array}{c}-0.3 \pm 0.49 \\
\text { small }\end{array}$ & $\begin{array}{c}-0.12 \pm 0.52 \\
\text { unclear }\end{array}$ & $\begin{array}{c}-0.07 \pm 0.05 \\
\text { trivial }\end{array}$ & $\begin{array}{c}-0.450 .49 \\
\text { small }\end{array}$ \\
\hline
\end{tabular}

Table 3: Cohen's d effect sizes. Abbreviations: CON - control intervention; LOW - Low-wrapping pressure intervention; HIGH - High-wrapping pressure intervention; PRE - assessment before intervention; POST - assessment after intervention; POST30 - assessment 30 minutes after intervention; ALSR - Active straight leg raise test; extMVC - knee extensors maximum voluntary contractions; flexMVC - knee flexors maximum voluntary contractions TcRF -rectus femoris muscle contraction time; TcBF -biceps femoris muscle contraction time; TcVM -vastus medialis muscle contraction time; DmRF -rectus femoris muscle displacement; DmBF -biceps femoris muscle displacement; DmVM - vastus medialis muscle displacement; $95 \% \mathrm{CI}-95 \%$ confidence interval.

\section{Discussion}

The aim of this study was to investigate the acute effects of wrapping floss band around the upper-thigh, while using different degrees of wrapping pressure, on ASLR, MVC, and TMG contractile characteristics of the knee extensors and flexors.

The findings of the present study show a statistically significant improvement in knee extensors MVC up to 30 minutes after the intervention (resulting in small to medium benefit) and a significant shortening in RF TMG contraction time (Tc_RF) immediately after the intervention (small benefit), where the LOW wrapping pressure condition was applied. In addition, medium benefits were associated with the LOW condition compared to HIGH for extMVC and Tc_RF, and medium benefits also discovered for RF displacement (Dm_RF) in the LOW condition compared to CON immediately after the floss application. Despite there being no statistically significant differences when CON and HIGH wrapping pressures were applied, there were medium benefits in BF displacement (Dm_BF) associated with the HIGH condition compared to CON. The results show an acute improvement in neuromuscular performance, particularly after the LOW condition compared to CON and HIGH conditions.

To the best of our knowledge, this is the first study investigating the effects of tissue flossing on MVC or other neuromuscular parameters, when the floss band was wrapped around the thigh. However, similar short-term benefits for tissue flossing applied over the ankle joint on athletic performance in recreationally trained individuals were recently reported; ${ }^{1}$ demonstrated a significant increase in single-leg vertical jump test after only 2 minutes of floss band application; in addition, ${ }^{3}$ observed an increase in countermovement 
jump force and sprinting time up to 45 minutes after flossing. In contrast, tissue flossing seems to be less effective when applied to elite athletes ${ }^{19}$.

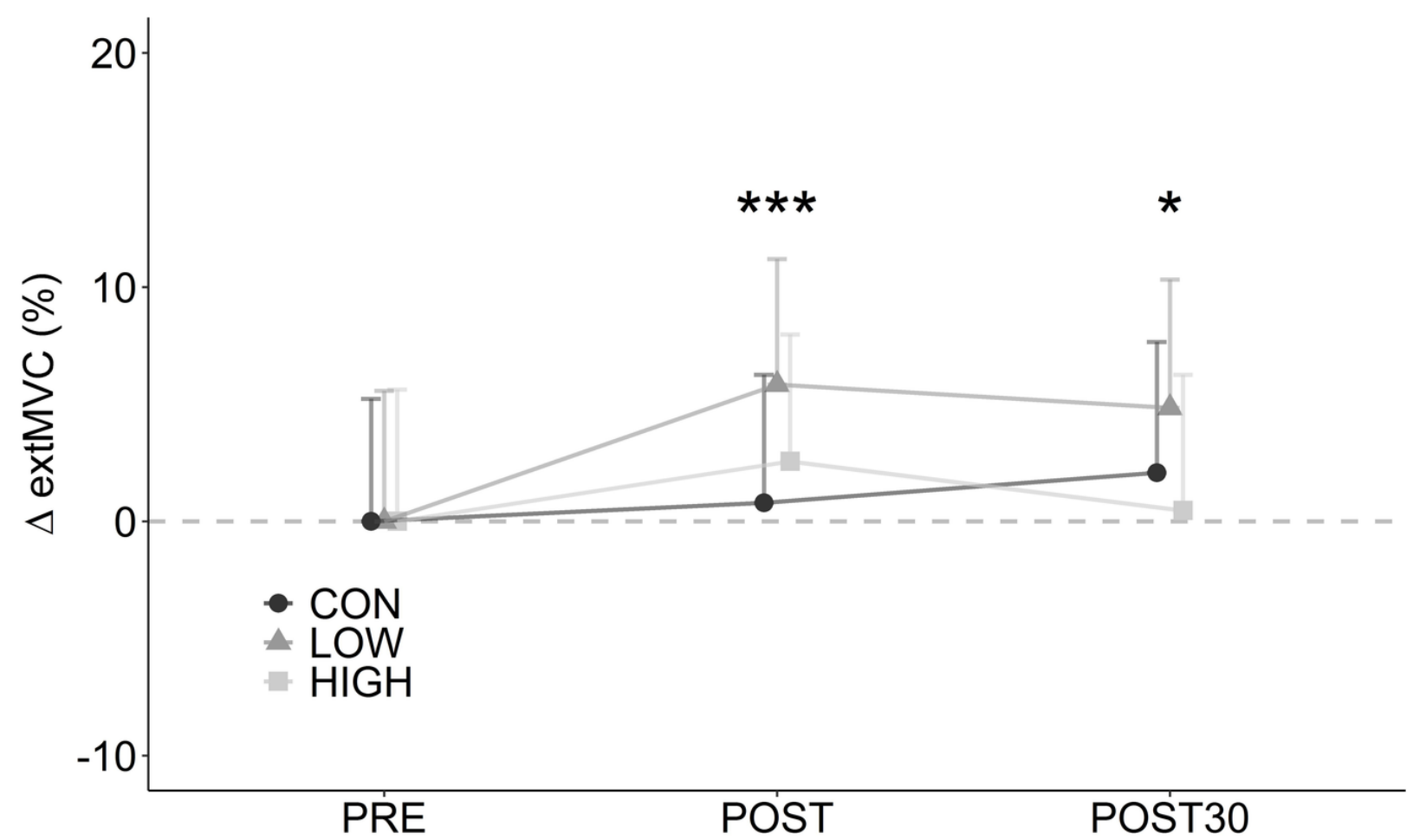

Figure 3: Relative changes in MVC from baseline for knee extensors: Statistically significant differences from baseline are represented by asterisks: ${ }^{*} \mathrm{p}<0.05 ;{ }^{* *} \mathrm{p}<0.01 ; * * * \mathrm{p}<0.001$.

Despite the lack of scientific evidence, the effects of tissue flossing have been identified as having similar effects of BFR ${ }^{1}$. In contrast to the findings of the present study, where limb wrapping induced neuromuscular potentiation (increased knee extensors MVC and faster RF muscle contraction time), studies investigating the acute effects of BFR on neuromuscular parameters showed significant muscle fatigue ${ }^{13,15,24,25}$. The observed discrepancies between the present study and those BFR studies may be associated with differences in intervention protocols. The active knee extension-flexion protocol without additional load adopted in the present study is similar to the protocols used in other tissue flossing studies ${ }^{3,19}$. In contrast, studies investigating the acute effects of BFR adopted protocols with several sets using additional load, ranging from $20 \%{ }^{25}$ up to $70 \% 1 \mathrm{RM}{ }^{15}$ to (or near to) volitional muscle failure. ${ }^{24}$ observed drops in MVC, level of voluntary activation, potentiated single twitch and EMG amplitude after a fatiguing protocol using BFR.

Similarly, ${ }^{13}$ reported a substantial drop in neuromuscular performance during a fatiguing protocol using BFR. However, all the measured parameters recovered to near baseline levels within 2 minutes after cuff removal. Tissue reperfusion has been proposed as the primary mechanism for neuromuscular parameter recovery in BFR studies ${ }^{13,26}$.

According to studies where the acute effects of BFR on neuromuscular performance were investigated, the observed fatigue seems to be affected by wrapping pressure, where it has been suggested that higher pressures can induce considerable change in neuromuscular parameters ${ }^{18,25} .{ }^{25}$ investigated the acute effects of BFR on 
MVC and EMG amplitude using different degrees of wrapping pressure. The authors observed a pressuresensitive decrease in MVC immediately following BFR intervention and an increase in EMG during the fatiguing protocol (higher pressures resulting in more significant parameter shift). However, 1 minute after cuff removal, MVC and EMG recovered to baseline levels. Similarly, ${ }^{18}$ indicated that neuromuscular fatigue, as well as muscle activation, are pressure-sensitive and vary as a function of relative vascular occlusion. In contrast, ${ }^{15}$ were unable to find any significant difference between local (BFR) and systemic hypoxic stimuli, or a combination of both, on neuromuscular performance, regardless of the degree of wrapping pressure. Compared to the findings identified in the previously mentioned BFR studies, in the present study, only the LOW condition induced feasible evidence of neuromuscular potentiation, suggesting that individualized pressure should be used in order for neuromuscular potentiation to take place.

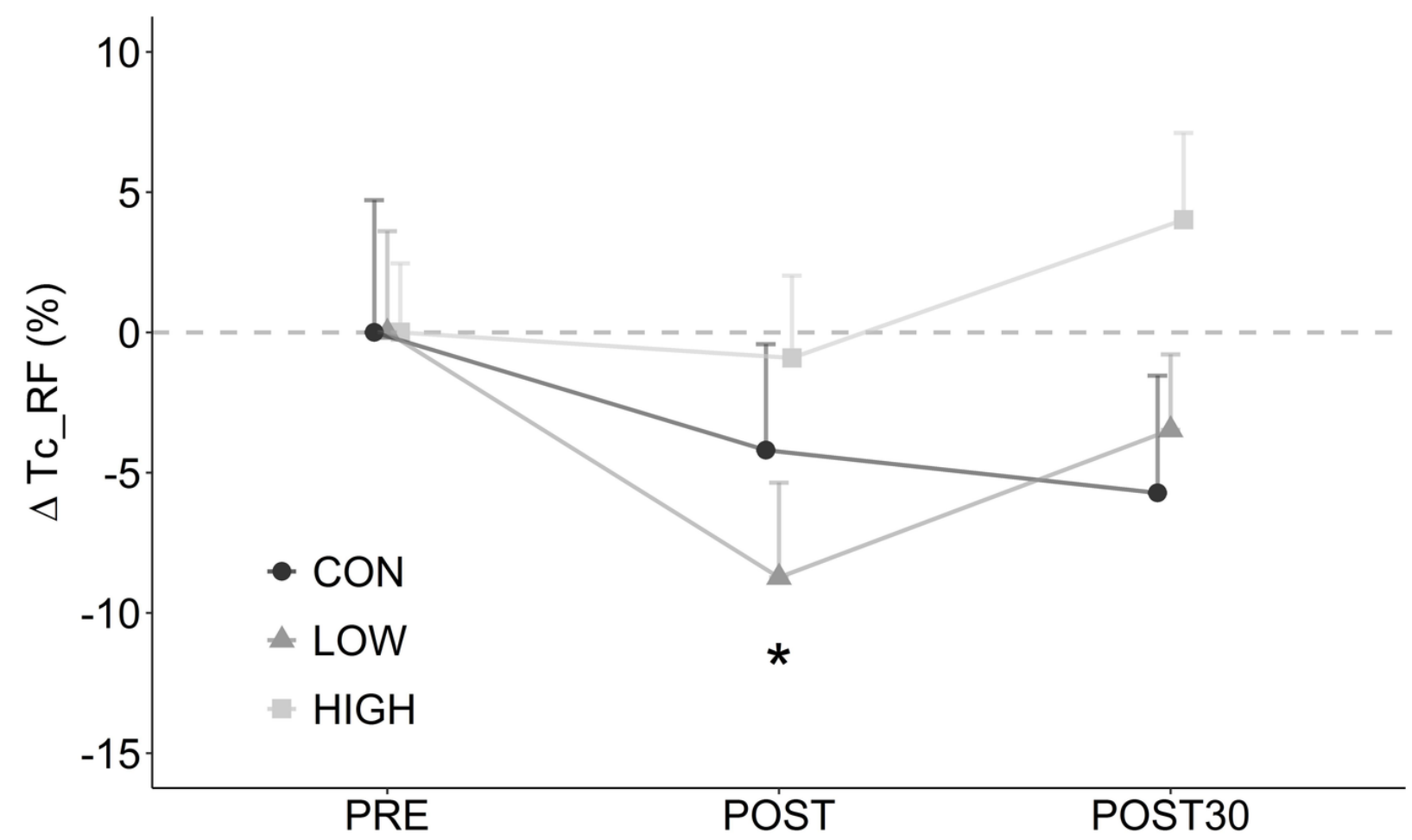

Figure 4: Relative changes in TMG contraction time (Tc) from baseline for RF muscle. Statistically significant differences from baseline are represented by asterisks: ${ }^{*} \mathrm{p}<0.05$; ${ }^{* *} \mathrm{p}<$ $0.01 ; * * * \mathrm{p}<0.001$.

In essence, tissue flossing creates conditions that are comparable to localized tissue hypoxia, where muscles need to adapt to a lack of oxygen. Although the partial vascular occlusion stimuli used in this study are substantially shorter ( $3 \times 3$ minutes) compared to systemic acute hypoxia stimulation, which is usually up to 40 minutes long, there is some evidence that systemic hypoxia actualized via hypoxic chambers can produce similar physiological responses to BFR training ${ }^{27}$. It has been suggested that the excitation of the sarcolemma remains unaffected by systemic hypoxia ${ }^{28,29}$. However, in a study where TMG parameters were used to assess muscle contractile function at acute moderate altitude (hypoxia), an increment of Tc and decrement of Dm was registered ${ }^{30}$. In a recent study, ${ }^{2}$ investigated the acute effects of a floss band applied over the ankle joint on the TMG parameters of the gastrocnemius medialis muscle but failed to find any statistically significant difference in TMG response. The authors suggested that tissue flossing has a 
significant but localized effect on the wrapped part of the body.

Similarly, the results of the present study show a significant shortening in RF contraction time (Tc_RF) immediately after tissue flossing intervention in the LOW condition and a medium effect in Dm_RF in favor of the LOW condition compared to CON and HIGH. At the same time, TMG parameters remained virtually unaltered in other muscles (VM and BF) in the CON and HIGH conditions. As proposed by ${ }^{31}$, a shortening Tc and a decrement in Dm parameters can be interpreted as a sign of neuromuscular potentiation. Since potentiation was only observed in RF after the LOW condition, it could be demonstrating that the effect of tissue flossing is precisely localized to the wrapped body area and has a negligible effect on the neuromuscular parameters of muscles distal from the wrapped area.

Moreover, in the present study, the ASLR test remained unaltered, regardless of the wrapping pressure. Even though there were no statistically significant differences, a drop in ASLR 30 minutes after the CON condition compared to LOW is very likely associated with themedium effect recorded. One of the most significant practical implications arising from previous tissue flossing studies where floss band had been applied around the ankle joint, was the enhancement in ankle joint range of motion (ROM) while maintaining, or even increasing, athletic performance ${ }^{1,3}$. In contrast, the findings of the present study suggest that when floss band is wrapped around the upper thigh, it has no significant effect on the adjacent joint range of motion (ASLR test remained unchanged). It could suggest that hypoxia and reperfusion may not be the only mechanism inducing ROM improvement after tissue flossing. These findings suggest that there are different mechanisms causing the observed changes in neuromuscular parameters. We can speculate that one possible mechanism could be the stimulation of pressure-sensitive mechanoreceptors in the tissue directly under the wrapped part of the body. The fact that mechano-sensory receptors are more abundant in ankle joint tissue compared to muscles and tendons ${ }^{32}$ may explain why ROM improved in ankle wrapping studies but no ASLR improvement was observed in the present study.

The present study might have some limitations. First: although the pressure was individualized according to the literature and controlled using a pressure sensor, there was no insight into the actual blood-flow occlusion created in the affected leg. Furthermore, it was difficult to consistently and accurately apply the floss band in the HIGH condition, resulting in higher deviation from the target pressure. Second: it is difficult to explain why knee flexors were not affected by the floss band since they were partially wrapped as well. Although the floss bands were expertly applied for consistent banding on the front and back of the thigh, pressure sensors were only placed frontally therefore pressure, and thus vascular occlusion, for BF may have been different.

\section{Conclusions}

Based on the findings of this study, we conclude that the observed neuromuscular potentiation was induced by the relative pressure of floss band wrapping, with a submaximal individualized wrapping pressures producing the best results. In addition, tissue flossing might have a significant, yet localized, effect on the specific body part being treated. Further research is needed to investigate how tissue flossing controls vascular blood flow occlusion using equipment such as a Doppler probe.

\section{Acknowledgement}

We want to thank all the participants in the study and members of the institute, who helped execute the research. 


\section{References}

1. Driller MW, Overmayer RG. The effects of tissue flossing on ankle range of motion and jump performance. Physical Therapy in Sport. 2017;25:20-24.

2. Vogrin M, Novak F, Licen T, Greiner N, Mikl S, Kalc M. Acute Effects of Tissue Flossing on Ankle Range of Motion and Tensiomyography Parameters. Journal of Sport Rehabilitation. 2020:1-7.

3. Driller M, Mackay K, Mills B, Tavares F. Tissue flossing on ankle range of motion jump and sprint performance: A follow-up study. Physical Therapy in Sport. 2017;28:29-33.

4. Prill R, Schulz R, Michel S. Tissue flossing: a new short-term compression therapy for reducing exerciseinduced delayed-onset muscle soreness. A randomized controlled and double-blind pilot crossover trial. The Journal of Sports Medicine and Physical Fitness. 2019;59(5).

5. Gorny V, Stöggl T. Einfluss von Flossing auf die Regenerationsfähigkeit der unteren Extremität nach Kraftausdauer-Belastungen. Sportverletzung · Sportschaden. 2018;32(01):55-60.

6. Loenneke JP, Pujol TJ. The Use of Occlusion Training to Produce Muscle Hypertrophy. Strength and Conditioning Journal. 2009;31(3):77-84.

7. Takano H, Morita T, Iida H, et al. Hemodynamic and hormonal responses to a short-term low-intensity resistance exercise with the reduction of muscle blood flow. European Journal of Applied Physiology. 2005;95(1):65-73.

8. Fahs CA, Loenneke JP, Thiebaud RS, et al. Muscular adaptations to fatiguing exercise with and without blood flow restriction. Clinical Physiology and Functional Imaging. 2014;35(3):167-176.

9. Loenneke JP, Thiebaud RS, Abe T. Does blood flow restriction result in skeletal muscle damage? A critical review of available evidence. Scandinavian Journal of Medicine 83 Science in Sports. 2014;24(6):e415-422.

10. Scott BR, Loenneke JP, Slattery KM, Dascombe BJ. Exercise with Blood Flow Restriction: An Updated Evidence-Based Approach for Enhanced Muscular Development. Sports Medicine. 2014;45(3):313-325.

11. Takarada Y, Sato Y, Ishii N. Effects of resistance exercise combined with vascular occlusion on muscle function in athletes.. Eur J Appl Physiol. 2002;86:308-14.

12. COOK SUMMERB, MURPHY BETHANYG, LABARBERA KATHERINEE. Neuromuscular Function after a Bout of Low-Load Blood Flow-Restricted Exercise. Medicine 83 Science in Sports 83 Exercise. 2013;45(1):67-74.

13. HUSMANN FLORIAN, MITTLMEIER THOMAS, BRUHN SVEN, ZSCHORLICH VOLKER, BEHRENS MARTIN. Impact of Blood Flow Restriction Exercise on Muscle Fatigue Development and Recovery. Medicine 8 Science in Sports \& Exercise. 2018;50(3):436-446.

14. Sieljacks P, Matzon A, Wernbom M, Ringgaard S, Vissing K, Overgaard K. Muscle damage and repeated bout effect following blood flow restricted exercise. European Journal of Applied Physiology. 2015;116(3):513-525.

15. Girard O, Willis SJ, Purnelle M, Scott BR, Millet GP. Separate and combined effects of local and systemic hypoxia in resistance exercise. European Journal of Applied Physiology. 2019;119(10):2313-2325.

16. Scott BR, Slattery KM, Sculley DV, Dascombe BJ. Hypoxia and Resistance Exercise: A Comparison of Localized and Systemic Methods. Sports Medicine. 2014;44(8):1037-1054.

17. Loenneke JP, Kim D, Fahs CA, et al. Effects of exercise with and without different degrees of blood flow restriction on torque and muscle activation. Muscle $\&$ Nerve. 2015;51(5):713-721. 
18. Fatela P, Reis JF, Mendonca GV, Avela J, Mil-Homens P. Acute effects of exercise under different levels of blood-flow restriction on muscle activation and fatigue. European Journal of Applied Physiology. 2016;116(5):985-995.

19. Mills B, Mayo B, Tavares F, Driller M. The Effect of Tissue Flossing on Ankle Range of Motion Jump, and Sprint Performance in Elite Rugby Union Athletes. Journal of Sport Rehabilitation. 2020;29(3):282-286.

20. Brophy-Williams N, Driller MW, Halson SL, Fell JW, Shing CM. Evaluating the Kikuhime pressure monitor for use with sports compression clothing. Sports Engineering. 2013;17(1):55-60.

21. Tous-Fajardo J, Moras G, Rodríguez-Jiménez S, Usach R, Doutres DM, Maffiuletti NA. Inter-rater reliability of muscle contractile property measurements using non-invasive tensiomyography. Journal of Electromyography and Kinesiology. 2010;20(4):761-766.

22. Šimunič B. Between-day reliability of a method for non-invasive estimation of muscle composition. Journal of Electromyography and Kinesiology. 2012;22(4):527-530.

23. Batterham AM, Hopkins WG. Making meaningful inferences about magnitudes.. Int J Sports Physiol Perform. 2006;1:50-7.

24. Karabulut M, Cramer JT, Abe T, Sato Y, Bemben MG. Neuromuscular fatigue following low-intensity dynamic exercise with externally applied vascular restriction. Journal of Electromyography and Kinesiology. 2010;20(3):440-447.

25. Yasuda T, Brechue WF, Fujita T, Sato Y, Abe T. Muscle activation during low-intensity muscle contractions with varying levels of external limb compression.. J Sports Sci Med. 2008;7:467-74.

26. COOK SUMMERB, CLARK BRIANC, PLOUTZ-SNYDER LORIL. Effects of Exercise Load and Blood-Flow Restriction on Skeletal Muscle Function. Medicine 83 Science in Sports 83 Exercise. 2007;39(10):1708-1713.

27. Schoenfeld BJ. Potential Mechanisms for a Role of Metabolic Stress in Hypertrophic Adaptations to Resistance Training. Sports Medicine. 2013;43(3):179-194.

28. Kayser B, Bkenkamp R, Binzoni T. Alpha-motoneuron excitability at high altitude. European Journal of Applied Physiology and Occupational Physiology. 1993;66(1):1-4.

29. Rupp T, Racinais S, Bringard A, Lapole T, Perrey S. Modulation of exercise-induced spinal loop properties in response to oxygen availability. European Journal of Applied Physiology. 2014;115(3):471-482.

30. Morales-Artacho AJ, Padial P, Rodríguez-Matoso D, et al. Assessment of Muscle Contractile Properties at Acute Moderate Altitude Through Tensiomyography. High Altitude Medicine 85 Biology. 2015;16(4):343-349.

31. Wilson MT, Ryan AMF, Vallance SR, et al. Tensiomyography Derived Parameters Reflect Skeletal Muscle Architectural Adaptations Following 6-Weeks of Lower Body Resistance Training. Frontiers in Physiology. 2019;10.

32. Taylor JL. Proprioception. In: Encyclopedia of Neuroscience. Elsevier; 2009:1143-1149. 